\title{
Threshold Algorithm For Pancreas Segmentation in Dixon Water Magnetic Resonance Images
}

\author{
Xiaoying Shan, Chaolin Du, Yufei Chen \\ College of Electronic and Information Engineering, \\ Tongji University, \\ Shanghai, China \\ Xiaoliang Gong \\ College of Electronic and Information Engineering, \\ Tongji University, \\ Shanghai, China \\ The Key Laboratory of Embedded System and Service \\ Computing, Ministry of Education, Tongji University, \\ Shanghai, China
}

\begin{abstract}
Pancreas segmentation is crucial for a computer-aided diagnosis (CAD) system to provide cancer detection and radiation therapy of pancreatic cancer. Because of anatomically high-variability between subjects, achieving high accuracies in pancreas segmentation remains a challenging task. In this work, based on Otsu threshold method and morphological method, we first proposed a segmentation pipeline for pancreas, using Dixon water magnetic resonance image (MRI) data from five healthy volunteers. The threshold method was used to obtain the approximate outline of the pancreas, and the morphological method was used to separate the pancreas from the surrounding tissues. The segmentation results were compared with manual contours using Dice Index (DI) and we achieved DI: $0.80 \pm 0.08$ which was better than the level Set Methods (LSMs) DI: 0.64 \pm 0.08. The proposed method was simple and easy to integrate with the Medical Imaging Interaction Toolkit (MITK) workbench, so it provided an efficient and simple segmentation method for processing large clinical datasets.
\end{abstract}

Keywords-component; Pancreas segmentation; Dixon water magnetic resonance image; Otsu method; morphological method

\section{INTRODUCTION}

Pancreatic adenocarcinoma is one of the deadliest cancers in the world. Accurate segmentation of the pancreas can be crucial for computer aided diagnosis that provide cancer detection and radiation therapy of pancreatic cancer. Automatic segmentation of numerous organs have been well studied with good performance for organs such as liver, stomach or kidneys, where Dice coefficients (DI) of > 0.9 were achieved [1-4]. However, achieving high accuracy in pancreas segmentation remains a challenging task. The shape, size and location of the pancreas differ greatly between subjects and visceral fat around the pancreas can drastically vary the boundary contrast as well. Previous segmentation works reported only 0.66 to 0.82 DIs [1$2,5]$. In [5], Shimizu et al. developed an automated pancreas segmentation algorithm. The algorithm used spatial

\author{
Asoke Nandi \\ Department of Electronic and Computer Engineering, \\ Brunel University \\ London, UK \\ College of Electronic and Information Engineering, \\ Tongji University, \\ Shanghai, China
}

Chao Ma, Panpan Yang

Department of Radiology, Changhai Hospital of Shanghai, Shanghai, China

standardization of pancreas and patient specific probabilistic atlas to cope with the variability in location and shape of the pancreas. Then a classifier ensemble was used to refine the rough segmentation results. The average Jaccard index between a true pancreas and the extracted one was 0.579 for 20 subjects. In [2], Shen et al. proposed a fully automated algorithm for abdominal organs using a multi-atlas-based segmentation method and morphological operations. The accuracy of pancreas segmentation represented by DI was $0.672 \pm 0.155$ based on 40 MRI datasets. In [1], Gou et al. used a hybrid gradient, region growth and shape constraint method to segment two-dimensional (2D) upper abdominal dynamic MRI images which were acquired from two patients. Dice coefficient was up to 0.82 . Although the accuracy of pancreas segmentation in [1] was superior to the above methods, the segmentation method was tested on only two healthy volunteers.

Image threshold is one of the most effective segmentation methods. In the past decades, many threshold techniques have been presented for medical image analysis [16-18]. The image processing operators based on classical mathematical morphology [7] has been widely used in medical image analysis and processing [19]. In recent studies, some advanced morphological processing operators have been proposed and it is used in medical image processing, such as blood vessel segmentation [20-21].

In this work, we proposed an automated algorithm based on the Otsu threshold method [6] and a morphological method. We first proposed a segmentation pipeline for pancreas segmentation, using Dixon water magnetic resonance image (MRI) data from five healthy volunteers, then calculated the similarity between the results from this pipeline and the manual contours using DI. The segmentation results was compared with the Level Set Method. 


\section{MATERIALS AND METHODS}

\section{A. MRI acquisition}

The MRI images were collected from 2 female and 3 male subjects whose average age is 52.6. The abdominal regions of the subjects were scanned on a 3T scanner (MAGNETOM Skyra, Siemens, Erlangen, Germany) with an 18-channel body receiver coil. Axial Dixon images were acquired with breath hold (14 seconds). Acquisition parameters were: $\mathrm{TR}=3.97 \mathrm{~ms}$, $\mathrm{TE}=1.26 / 2.49 \mathrm{~ms}$, flip angle $=9^{\circ}, 332 \times 240$ acquisition matrix size, FOV $=400 \times 325.2 \mathrm{~cm}^{2}$, reconstruction slice thickness $=3$ $\mathrm{mm}$, 64 slices, spacing between slices $=0 \mathrm{~mm}$. A built-in software was used to reconstruct the Dixon water images. The reconstructed MRI data had 40 slices for each subject. Manual reference segmentation was performed by a trained physician by manually delineating the contours of pancreas on all 40 slices, which are taken as the ground truth.

\section{B. Segmentation Methods}

In our work, image threshold was adopted for pancreas image segmentation. Because of its intuitive properties and simplicity of implementation, image threshold plays an important role in image segmentation [8-10]. Its basic objective is to divide a given image into two classes: foreground and background. Otsu method is an attractive method for optimal global threshold processing. By choosing the threshold value $k$ to maximize the variance between foreground and background, the new image $f(x, y)$, a binary image, is defined as

$$
f(x, y)=\left\{\begin{array}{l}
1 \quad \text { if } g(x, y) \geq k \\
0 \quad \text { if } g(x, y)<k
\end{array}\right.
$$

Mathematical morphology is a useful tool for extraction of image composition and image preprocessing or post-processing, such as morphological filtering, thinning, and pruning. In order to achieve the purpose of image analysis and recognition, Mathematical morphology uses some structuring elements to measure and extract the corresponding shape of image. Dilation and erosion are two basic morphological operations. Erosion is an operation that "shrinks" foreground in a binary image and dilation thickens foreground in a binary image. A structuring element is used to control the specific manner and degree of shrinking or thickening. In mathematics, dilation and erosion are defined in term of set operations. The erosion of $\mathrm{A}$ by $\mathrm{B}$, denoted as $A \ominus B$, is defined as

$$
A \ominus B=\left\{\mathrm{z} \mid B_{z} \subseteq A\right\}
$$

where set $\mathrm{A}$ be a set in $\mathrm{Z}^{2}$ and $\mathrm{B}$ is a structuring element. Essentially, the erosion of A by B is the collection of the origin positions of all structural elements, where the conversion B does not overlap with the background of the A. The dilation of A by $\mathrm{B}$, denoted as $A \oplus B$, is defined as

$$
\left.A \oplus B=\{\mathrm{z} \mid \hat{(B})_{z} \cap A \neq \varnothing\right\}
$$

where $\varnothing$ is the empty set. In other words, the dilation of A by $\mathrm{B}$ is the set consisting of all the structuring element origin locations where the reflected and translated $B$ has overlap at least some parts of A. Erosion can remove areas of an object that do not contain the structuring element, break thin connections, removes thin protrusions and smooth object outline. Dilation can join narrow breaks, fill up holes smaller than the structuring element and smooth the object contours by filling up narrow gulfs. In image processing applications, the operations of dilation and erosion are most commonly used in a variety of combinations. In our pancreas segmentation algorithm, an image underwent erosion twice and then dilations twice using the same structuring elements. The aims of morphological operation were to isolate different organs, remove the adhesion of organs and to fill holes in the binary images.

The proposed algorithm (TM) is based on the Otsu threshold method [6] and a morphological method. It was integrated with the Medical Imaging Interaction Toolkit (MITK) workbench. MITK is a free open-source software system for development of interactive medical image processing software. The algorithm pipeline was implemented as follows:

1) Import MRI data into MITK workbench.

2) Select regions of interest. Subsequently, the following operations were performed on the regions of interest.

3) Use Otsu method for segmentation of the images which resulted in binary images.

4) Perform erosion operation twice and then dilation operation twice on the binary images.

5) Select the region of pancreas, then remove the remaining parts.

6) Three dimensional reconstruction of pancreas using the segmentation results.

\section{Comparing Methods}

The level set methods (LSMs) presented by Osher and Sethian [11] have been proven to be one of the most successful methods for image segmentation. The basic idea of level set methods is to represent the curves or surfaces as level sets of higher dimensional functions so as to yield seamless processing of topological changes. Thus, an unknown number of objects can be detected at the same time without resorting to dedicated contour tracking. Generally speaking, the existing level set methods can be categorized into two classes: edge-based models [12-13] and region-based models [14-15]. The edgebased models drive the motion of the zero level set toward desired object boundaries by using image gradient as an additional constraint. Region-based models exploit the image statistical information inside and outside the contour to evolve a curve to extract the desired object. In [15], Li et al. presented an edge-based level set method named distance regularized level set (DRLS) for image segmentation. The resulting evolution of DRLS function is the gradient flow that minimizes the overall energy functional with a distance regularization term and an external energy that stop the contours on the desired object boundaries. In this study, initial contours were manually drawn on the first imaging slice and then propagated to the subsequent slices. Briefly, segmentation was performed based on a distance regularized level set energy function [15]: 


$$
\varepsilon(\phi)=\mu R_{p}(\phi)+\lambda L_{g}(\phi)+\alpha A_{g}(\phi)
$$

where $\phi$ was the level set function, $R_{p}(\phi)$ was the level set regularization term, $L_{g}(\phi)$ computed the line integral of the function along the zero level contour, and $A_{g}(\phi)$ was used to accelerate the motion of the zero level contour in the level set evolution process. The external energy, $L_{g}(\phi)$ and $A_{g}(\phi)$, drove the zero level set toward the object boundaries, while the internal energy $R_{p}(\phi)$ kept the evolving level set function as an approximate signed distance function during the evolution. In this study, we used the DRLS model for pancreas segmentation. We adopted the same parameter values for $\mu(0.2)$ and $\lambda$ (5) from the original publication, but the value for $\alpha$ resulted in poor segmentation performance. Therefore, we set $\alpha$ as $[2.5,4]$ by trial and error and adjusted $\alpha$ for individual subjects based on segmentation results.

\section{Validation of the segmentation}

In addition to the automated segmentation, manual reference segmentation was performed by a trained physician on MITK workbench by manually delineating the contours of

$$
\text { Original images Manual }
$$
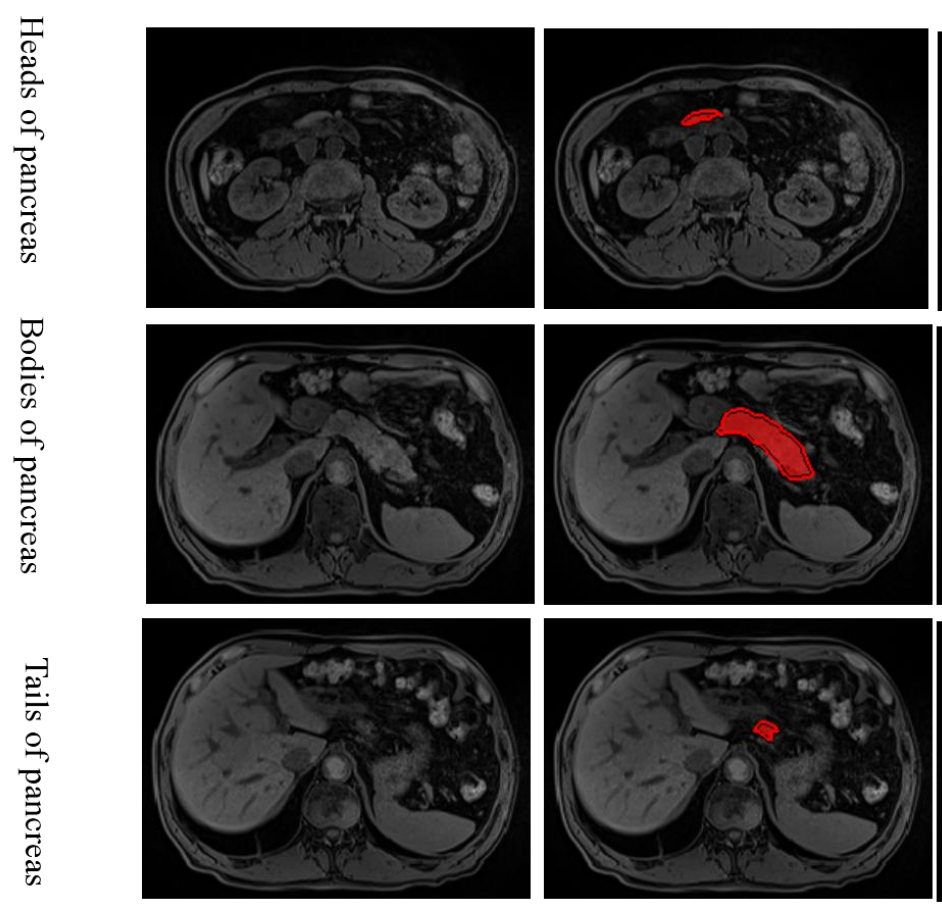
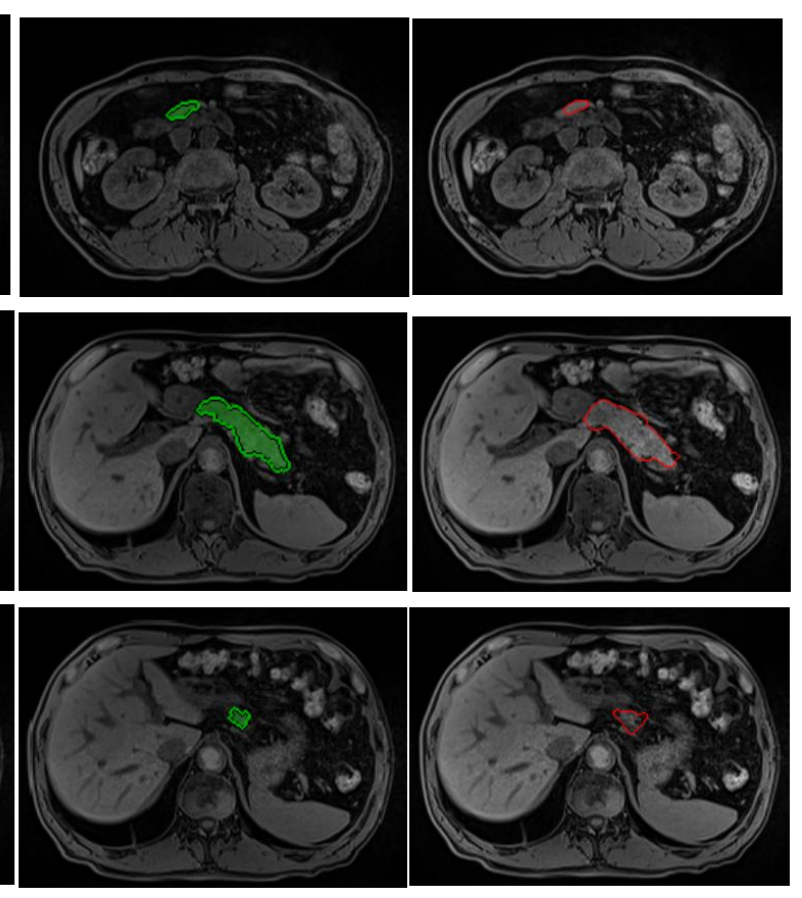

pancreas on all slices. The segmentation results were evaluated both visually and quantitatively.

To quantitatively analyze the segmentation performance, Dice coefficients was calculated using

$$
D I=2 \frac{\left|A_{1} \cap A_{2}\right|}{\left|A_{1}\right|+\left|A_{2}\right|},
$$

where $A_{1}$ and $A_{2}$ were the binary masks from automated and manual segmentation, respectively.

\section{RESULTS AND DISCUSSION}

Figure 1 showed the segmentation results of three selected imaging slices from the same subject. The TM results agreed well with manual segmentation results visually despite the low conspicuity of organ boundaries and significant adhesion between pancreas and other organs. The results of DRLS method show under- or over-segmentation for the pancreas. The reason was that the zero level sets stopped the contours on the boundaries of other organs or tissues which were very near the pancreas, while the zero level set crossed the weak boundaries of pancreas.

\section{$\mathrm{TM}$}

\section{DRLS}

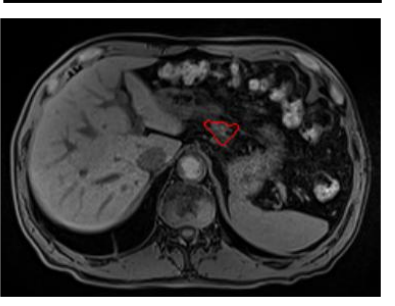

Figure 1. Segmentation comparison for different imaging techniques. Automated segmentation results and the manual segmentation results were shown as superimposed contours.

The segmentation performance was quantified for the two methods using the Dice index with reference to the manual segmentation (the ground truth). Table 1 showed the average DIs which were shown as mean \pm standard deviation. The average DI for each subject was computed by averaging the DIs of all slices. TM resulted in higher average DIs for all five subjects. From the view of the average DI value of all five subjects, the TM resulted in more accurate pancreas segmentation (DI $=0.80 \pm 0.08)$ than DRLS $(\mathrm{DI}=0.64 \pm 0.08)$. Moreover TM was more robust than DRLS.

MRI data for each subject contained 40 slices. The average DI for each layer was calculated by averaging DIs of the same 
layer for all five subjects. Figure 2 showed the average DIs for 40 layers. For each slice, the average DI from TM was higher than DRLS. Because of the low conspicuity of organ boundaries and significant adhesion between pancreas and other organs, the segmentation performance of TM and DRLS were both not good for the tail of pancreas. Figure 3 showed the DIs of TM segmentation results of all 40 slices for all five subjects. In some case (case 1 and 5), we could find the segmentation accuracy of TM were poor for the head of pancreas. The Figure 4 showed the 3D rendering of the pancreas contour based on the manual segmentation and TM segmentation respectively.

TABLE I. Average DI COMParison OF 2 Methods On All SLICES

\begin{tabular}{|l|c|c|}
\hline \multirow{2}{*}{ case } & \multicolumn{2}{|c|}{ Segmentation methods } \\
\cline { 2 - 3 } & DRLS & $\boldsymbol{T M}$ \\
\hline 1 & $0.66 \pm 0.11$ & $0.81 \pm 0.11$ \\
\hline 2 & $0.63 \pm 0.12$ & $0.84 \pm 0.06$ \\
\hline 4 & $0.67 \pm 0.07$ & $0.81 \pm 0.11$ \\
\hline 5 & $0.60 \pm 0.09$ & $0.78 \pm 0.14$ \\
\hline $\begin{array}{c}\text { Average DI of } \\
\text { all 5 subjects }\end{array}$ & $0.66 \pm 0.09$ & $0.81 \pm 0.11$ \\
\hline
\end{tabular}

DIs were shown as mean \pm standard deviation.

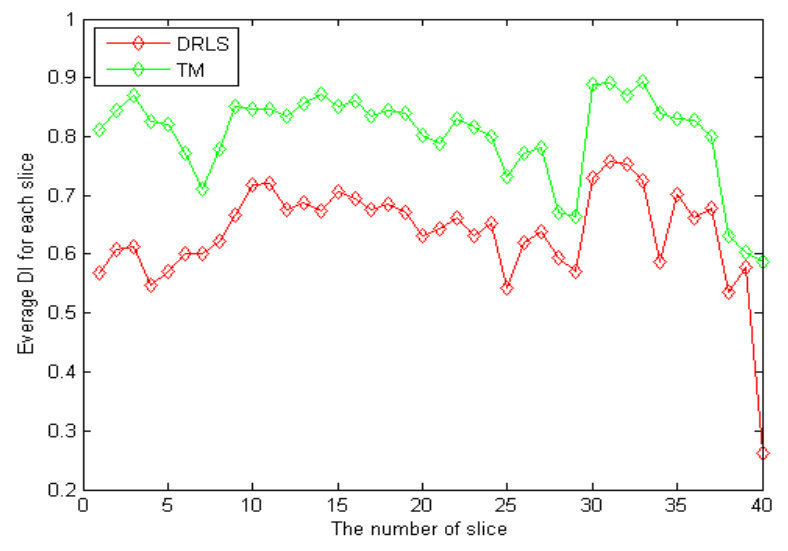

Figure 2 Comparison of the everage DIs for each slice

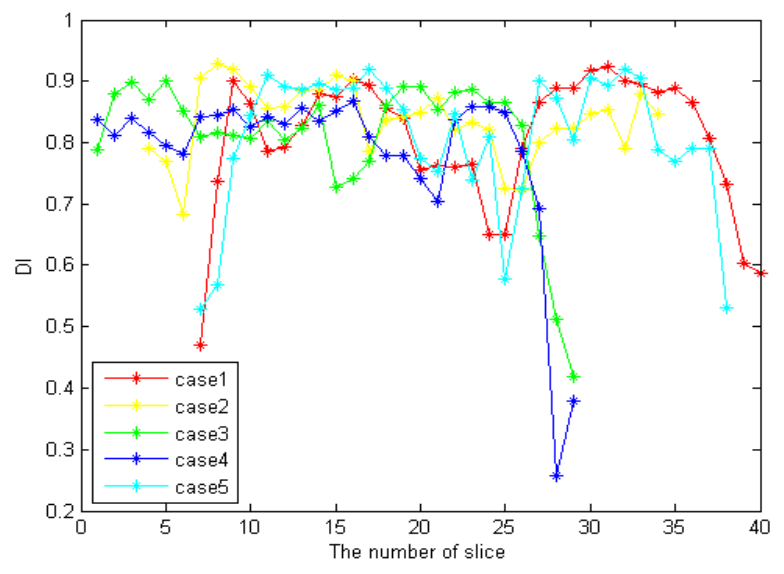

Figure 3 DIs of the results for the TM method

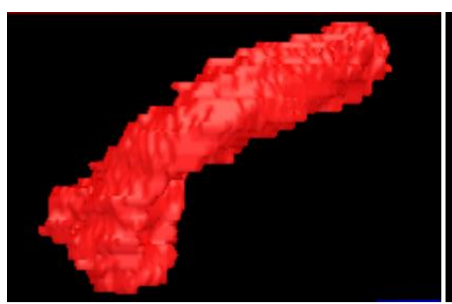

A

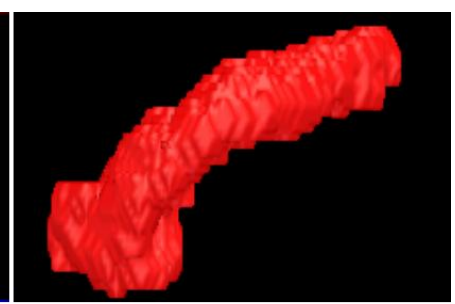

B
Figure 4 (A) A 3D rendering of the pancreas contour based on the manual results of case 2 (B) A 3D rendering of the pancreas contour based on the results of TM.

\section{CONCLUSION}

Pancreatic adenocarcinoma is one of the deadliest cancers in the world. Accurate segmentation of the pancreas can be crucial for computer aided diagnosis that provide cancer detection and radiation therapy of pancreatic cancer. In this paper, we proposed an automated algorithm based on Otsu method and morphological method (TM) for pancreas segmentation, using Dixon water MRI data from five healthy volunteers. The segmentation results were compared with manual contours using Dice coefficients and we achieved 0.80 Dice coefficient. The achieved accuracy was promising for pancreas localization. The proposed TM method was simple and easy to integrate with the Medical Imaging Interaction Toolkit (MITK) workbench, so it provided an efficient and simple segmentation method for doctors. There are several limitations in the study. First, every subject is healthy. When the subject was a healthy person or a patient who has diseasealtered morphology, whether the performance of segmentation method was significantly affected by the subject need to be tested. Second, more subjects are needed to show the robustness of segmentation method. In this paper, we also pointed out that the head of the pancreas and the tail of pancreas had poorer accuracy than the body of pancreas. In our future work, improving the segmentation accuracy of the head of a pancreas and the tail of a pancreas will be the aim of our research.

\section{ACKNOWLEDGMENT}

This work is supported by Shanghai Natural Science Foundation of china (no. 16JC1401300). The authors would like to thank Dr Chao Ma and Dr Panpan Yang of the Changhai Hospital of Shanghai for providing them with the Dixon water MR images as well as the manual pancreas segmentations.

\section{REFERENCES}

[1] S. Gou, P. Lee, P. Hu, JC. Rwigema, K. Sheng and P. Hu, et al.,"Feasibility of automated pancreas segmentation based on dynamic MRI," Journal of Radiology, British, vol 87, 20140248, September 2014.

[2] J. Shen, T. Baum, C. Cordes, B. Ott, T. Skurk andH. Kooijman, et al., "Automatic segmentation of abdominal organs and adipose tissue compartments in water-fat MRI: application to weight-loss in obesity," European Journal of Radiology, vol 85, pp. 1613-1621, August 2016.

[3] A. A. Joshi, H. H. Hu, R. M. Leahy, M. I. Goran, K. S. Nayak and K. S. Nayak, "Automatic intra-subject registration-based segmentation of abdominal fat from water-fat MRI," Journal of Magnetic Resonance Imaging, vol 37, pp. 423-430, February 2013. 
[4] R. Wolz, C. Chu, K. Misawa, K. Mori, D. Rueckert, "Multi-organ abdominal CT segmentation using hierarchically weighted subjectspecific atlases," International Conference on Medical Image Computing \& Computer-assisted Intervention, vol 15, pp.10-17, Med Image Comput Comput Assist Interv. October 2012.

[5] A. Shimizu, T. Kimoto, H. Kobatake, S. Nawano, K. Shinozaki, "Automated pancreas segmentation from three-dimensional contrastenhanced computed tomography," International Journal of Computer Assisted Radiology and Surgery, vol 5, pp. 85-98, January 2010.

[6] N. Otsu, "A threshold selection method from gray-Level histogram," IEEE Transactions on Systems Man \& Cybernetics vol 9, pp. 62-66, January 1979.

[7] R. C. Gonzalez and R. E. Woods. Digital Image Processing. Prentice Hall, January 2002.

[8] Y. B. Chen, T. C Chen, "Image segmentation method using thresholds automatically determined from picture contents," Eurasip Journal on Image \& Video Processing, vol. 1,pp. 1-15, January 2009.

[9] E. Navon, O. Miller, A. Averbuch, "A color image segmentation based on adaptive local thresholds," Image \& Vision Computing, vol. 23(1), pp. 69-85, January 2005.

[10] Q. B. Truong, B. R. Lee, "Automatic multi-thresholds selection for image segmentation based on evolutionary approach," International Journal of Control, Automation and Systems, vol. 11(4), pp. 834-844, August 2013.

[11] S. Osher, J. A. Sethian, "Fronts propagating with curvature-dependent speed: algorithms based on Hamilton-Jacobi formulations," Journal of Computational Physics, vol. 79(1), pp. 12-49, November 1988.

[12] T. F. Chan, L. A. Vese, "Active contours without edges," IEEE Transactions on Image Processing, vol. 10(2), pp. 266-277, February 2001.
[13] X. Wang, H. Min, Y. Zhang, "Multi-scale local region based level set method for image segmentation in the presence of intensity inhomogeneity," Neurocomputing, vol. 151, pp. 1086-1098, March 2015.

[14] A. Vasilevskiy, K. Siddiqi, "Flux-maximizing geometric flows," IEEE Transaction on Pattern Analysis and Machine Intelligence, vol. 24(12), pp. 1565-1578, December 2002.

[15] C. Li, C. Xu, C. Gui, and M. D. Fox, "Distance regularized level set evolution and its application to image segmentation," IEEE Transactions on Image Processing, vol. 19, pp. 3243-3254, December 2010.

[16] P. D. Sathya, R. Kayalvizhi, "Optimal segmentation of brain MRI based on adaptive bacterial foraging algorithm," Neurocomputing, vol. 74, pp. 2299-2313, July 2011.

[17] M. Maitra, A. Chatterjee, "A novel technique for multilevel optimal magnetic resonance brain image thresholding using bacterial foraging," Measurement, vol. 41, pp. 1124-1134, December 2008.

[18] S. Manikandan, K. Ramar, M. W. Iruthayarajan and K.G. Srinivasagan, "Multilevel thresholding for segmentation of medical brain images using real coded genetic algorithm," Measurement, vol. 47, pp. 558-568, January 2014.

[19] H. E. Cline, D. R. Thedens, C. H. Meyer, D.G. Nishimura, T. K. Foo et al., "Combined connectivity and a gray - level morphological filter in magnetic resonance coronary angiography," Magnetic Resonance in Medicine, vol. 43, pp. 892-895, June 2000.

[20] B. Bouraoui, C. Ronse, J. Baruthio, N. Passat and P. Germain, “3D segmentation of coronary arteries based on advanced mathematical morphology techniques," Computerized Medical Imaging \& Graphics, vol. 34, pp. 377-387, July 2010.

[21] A. Dufour, O. Tankyevych, B. Naegel, H. Talbot, C. Ronse et al., "Filtering and segmentation of 3D angiographic data: Advances based on mathematical morphology," Medical Image Analysis, vol. 17, pp. 147-164, February 2013. 